\title{
L'état du droit dans le transport routier de marchandises : une réglementation en trompe-l'œil
}

\section{Stéphane Carré}

\begin{abstract}
Condition of Law in Freight Road Transport : Deceptive Rules.

The freight road transport sector is in a paradoxical situation. It is a particularly regulated sector of activity. However, at the same time, professionals from this sector have not implemented numerous legal measures to which they were normally subjugated. So analysis of the entire set of rules cannot procure us a precise picture of the state of the law in this sector of activity. Indeed, the ineffectiveness of badly applied legal regulations seems to hide another legal reality, more flexible or different from how it might first appear.
\end{abstract}

\section{Résumé}

Le secteur des transports routiers de marchandises connaît une situation paradoxale. II s'agit d'un secteur d'activité particulièrement réglementé. Cependant, dans le même temps, les professionnels de ce secteur n'ont pas appliqué de nombreuses dispositions juridiques auxquelles ils étaient normalement assujettis. Aussi l'analyse de l'ensemble de ces règles ne peut-elle pas nous procurer un tableau précis de l'état du droit dans ce secteur d'activité. L'inanité d'une réglementation légale mal appliquée semble en effet cacher une autre réalité juridique, plus souple ou différente qu'elle n'y paraît de prime abord.

\section{Citer ce document / Cite this document :}

Carré Stéphane. L'état du droit dans le transport routier de marchandises : une réglementation en trompe-l'œil. In: Droit et société, n46, 2000. Complexités à l'œuvre. pp. 597-614;

doi : 10.3406/dreso.2000.1522

http://www.persee.fr/doc/dreso_0769-3362_2000_num_46_1_1522

Document généré le 06/06/2016 


\section{L'état du droit dans le transport routier de marchandises : une réglementation en trompe-l'œil}

\section{Stéphane Carré *}

\section{Résumé}

Le secteur des transports routiers de marchandises connaît une situation paradoxale. Il s'agit d'un secteur d'activité particulièrement réglementé. Cependant, dans le même temps, les professionnels de ce secteur n'ont pas appliqué de nombreuses dispositions juridiques auxquelles ils étaient normalement assujettis. Aussi l'analyse de l'ensemble de ces règles ne peut-elle pas nous procurer un tableau précis de l'état du droit dans ce secteur d'activité. L'inanité d'une réglementation légale mal appliquée semble en effet cacher une autre réalité juridique, plus souple ou différente qu'elle n'y paraît de prime abord.

Délégalisation - Droit des transports - Durée du travail - Effectivité - Normes juridiques - Ordre juridique.

\section{Summary}

\section{Condition of Law in Freight Road Transport : Deceptive Rules}

The freight road transport sector is in a paradoxical situation. It is a particularly regulated sector of activity. However, at the same time, professionals from this sector have not implemented numerous legal measures to which they were normally subjugated. So analysis of the entire set of rules cannot procure us a precise picture of the state of the law in this sector of activity. Indeed, the ineffectiveness of badly applied legal regulations seems to hide another legal reality, more flexible or different from how it might first appear.

Delegalisation - Law effectiveness - Legal norms - Legal order - Transport law - Working time.

\section{L'auteur}

Maître de conférences à l'IUT de Saint-Nazaire, membre du laboratoire " Droit et changement social ", Université de Nantes. Ses travaux portent principalement sur le droit des transports routiers. Outre la réalisation d'une thèse de doctorat en droit portant sur le contrôle par le conseil des prud'hommes des licenciements économiques, il a publié notamment :

- « La Cour de cassation face à

l'épineux contrôle de la légitimité du licenciement économique ", Le droit ouvrier, mars 1995 ;

— «Un exemple de pluralisme juridique : l'accord " Grands Routiers » du 23 novembre 1994 ", Revue juridique de l'Ouest, 3, 1999 ;

- Les transformations des relations du travail dans le transport routier de marchandises, I : La place du droit dans une entreprise de transport routier, rapport au ministère des Transports et de l'Équipement, 2000.

* Institut Universitaire de Technologie de Saint-Nazaire, Département Logistique et Transports,

Université de Nantes,

58, rue Michel Ange,

BP 420,

F-44606 Saint-Nazaire cedex. <stephane.carre@iutsn.univnantes.fr> 


\section{S. Carré}

L'état du droit dans le transport routier de marchandises : une réglementation en trompe-l'œil
1. P. HAMEUIN, « Réglementation du travail et pratiques : interrogations à propos d'un certain écart ", in Transports 93 : professions en devenir, Paris PENPC, 1992. Sur l'effectivité du droit en général : P. LASCOUMES et É. SERVERIN, "Théories et pratiques de l'effectivité du droit ", Droit et Société, 2, 1996 ; C. MINCKE, " Effets, effectivité, efficience et efficacité du droit : le pôle réaliste de la validité ", Revue interdisciplinaire d'études juridiques, 40, 1998 ; Ch. ATIAS et D. LINOTTE, " Le mythe de l'adaptation du droit au fait ", Recueil Dalloz, chronique XXXIV, 1977, p 251.

2. D. LARGER, « La conscience commune en droit du travail (encore quelques mots sur l'ineffectivité...) ", Droit social, 2, 1983.

3. L.M. FRIEDMAN, " La sociologie du droit est-elle vraiment une science ? ", Droit et Société, 2, 1986, p. 98.
Le secteur des transports routiers, et spécialement celui des marchandises, connait une situation paradoxale. Il s'agit, dès l'entre-deux-guerres, d'un secteur d'activité particulièrement réglementé. Cependant, dans le même temps, les professionnels ont su en pratique écarter l'application de nombreuses règles auxquelles ils étaient normalement assujettis, à tel point qu'un rapide aperçu des pratiques en usage dans la profession, jusqu'au milieu des années quatre-vingt-dix, donne parfois l'impression qu'aucune réglementation impérative n'existe ${ }^{1}$...

Pourtant, les entreprises de transports routiers doivent officiellement respecter un ensemble complexe de dispositions d'ordre public, parfois tout à fait spécifiques. En effet, ces entreprises doivent tenir compte de règles relevant du droit des transports, du droit du travail, du Sode de la route ou de la législation communautaire sur les temps de conduite.

L'" opinio juris ", cette conscience collective de l'utilité sociale des règles juridiques, semble donc faire singulièrement défaut aux membres de la profession ${ }^{2}$, et ce pour diverses raisons que nous aborderons : il est assurément possible de s'interroger sur les causes de cette ineffectivité.

Mais il est encore permis de s'interroger sur les conséquences de cette situation, relativement aux limites et à la consistance du droit a priori applicable. En effet, l'analyse de l'ensemble des règles édictées peut-elle nous procurer un tableau précis de l'état $d u$ droit dans ce secteur d'activité ? Il ne le semble pas. Le rapport au droit qu'entretiennent les différents acteurs de ce secteur est tel que le postulat sous-jacent d'une réglementation qui leur serait naturellement applicable ne peut être reçu sans d'importantes réserves. En d'autres termes, nous pouvons faire l'hypothèse, à partir de l'observation de cette situation particulièrement éclairante, que l'application des règles juridiques rétroagit, en quelque sorte, sur le droit lui-même 3 .

De la sorte, quelle réalité donner à une législation d'ordre public lorsque l'écart à la règle prend d'aussi importantes proportions ? De même, le droit applicable est-il simplement formulé à partir du contenu de dispositions légales et de décisions juridictionnelles quand sa place restreinte dans les relations sociales qui s'instaurent entre les différents acteurs de ce secteur d'activité s'avère en partie conditionnée par la législation elle-même? "L'inanité " d'une réglementation légale mal appliquée pourrait finalement cacher une autre réalité juridique, plus souple ou différente qu'elle n'y paraît de prime abord.

Il s'agit donc de reprendre une thèse classique, mais trop rarement illustrée à partir d'un exemple précis et concret, selon laquelle le droit positif, en tant que phénomène social, ne peut être limité à un simple ordonnancement juridique. Il serait plutôt la résultante de l'ensemble des pratiques et des représentations dé- 
coulant des normes juridiques. Cette assertion s'appuie sur la distinction effectuée par différents auteurs entre "l'ordonnancement juridique " d'une part, et le "droit ", le "système juridique " ou «l'ordre juridique » d'autre part.

Ainsi, Christian Atias considère que la norme juridique qui pourrait s'observer dans la société, par delà une collection de règles issues des différentes sources formelles du droit, naît des pratiques, des arbitrages, des appréciations de la population et des juristes ${ }^{4}$. Santi Romano rappelle l'existence d'ordres juridiques sans règle écrite ni même orale. Cet auteur identifie l'ordre juridique à une institution concrète, c'est-à-dire à une organisation sociale, les normes juridiques n'étant que le reflet de cette institution et le droit comprenant « les mécanismes et engrenages multiples, les rapports d'autorité et de force qui créent, modifient, appliquent, font respecter les normes juridiques sans s'identifier à celles-ci ${ }^{5}$.

En effet, jusqu'au milieu des années 1990, l'état du droit dans le secteur du transport routier de marchandises ébranle fortement l'image d'un système juridique fondé sur la prééminence d'une législation d'ordre public, d'application stricte. Outre la réglementation sociale, il existe pourtant de nombreuses règles impératives visant à organiser l'exercice de la profession et à assurer la sécurité sur le domaine public routier (règles sur les temps de conduite, Code de la route). Il s'agit alors de règles d'ordre public, au sens le plus strict ${ }^{6}$. Mais peu cohérent et de mise en cuvre difficile, l'encadrement juridique propre à ce secteur a des effets pernicieux qui gênent toute application stricte de la législation (I). Nous sommes plutôt en face d'un droit «mou ».

Cependant, ce n'est pas précisément cette incohérence qui est la cause première de l'inapplication du droit. Ces insuffisances viennent plutôt redoubler une difficulté récurrente à encadrer juridiquement les pratiques, pour des raisons sociologiques et économiques. Dès lors, ces insuffisances sont plutôt le reflet de cette difficulté et ne doivent rien au hasard d'une malencontreuse impéritie du législateur. Contrainte ou voulue, une certaine "politique juridique " s'est mise en place qui autorise un important écart à la loi. Cette politique juridique est non seulement l'activité législative grâce à laquelle le législateur entend atteindre certains buts (politique législative), mais encore, par exemple, l'activité administrative par laquelle l'État entend peser sur l'application des règles qu'il pose.

Du fait de cet important écart à la loi, le caractère d'ordre public de cette législation semble donc factice, comme de façade. En quelque sorte, nous serions en face d'un encadrement juridique permissif (II).
4. C. ATIAS, Épistémologie juridique, Paris, PUF, 1985, p. 51 ; ID., "Quelle positivité ? Quelle notion de droit?, Archives de philosophie du droit, tome 27, 1982.

5. S. ROMANo, L'ordre juridique [1946], Paris, Dalloz, 1975, p. 14 et 10 .

6. G. LYON-CAEN, « Négociation collective et législation d'ordre public », Droit social, 2, 1973 ; L. ROZES, " Remarques sur l'ordre public en droit du travail ", Droit social, 9/10, 1977. 


\section{S. Carré}

L'état du droit dans le transport routier de marchandises : une réglementation en trompe-l'œil

7. A. SUPIOT, Critique du droit du travail, Paris PUF, 1994, p. 212. Le terme " déjuridicisation " est encore employé (cf. « Dérégulation ", in A.-J. ARNAUD [sous la dir.], Dictionnaire encyclopédique de théorie et de sociologie du droit, Paris, LGDJ, 1993) malgré sa polysémie (A. JEAMMAUD, "Introduction à la sémantique de la régulation juridique. Des concepts en jeu ", in J. CLAM et G. MARTIN [sous la dir.], Les transformations de la régulation juridique, Paris, LGDJ, 1998, p. 47 et suiv.).

8. J. CARBONNIER, Flexible droit, Paris, LGDJ, 1983, p. 29.

9. B. LEFEBVRE, «Espaces professionnels et flux tendus ", Actes de la recherche en sciences sociales, 114, 1996.

10. Sur l'ineffectivité du droit du fait des incohérences et des imprécisions qu'il recèle, $c f$.

D. LARGER, 1983, art. cité, p. 109 ; F. RANGEON, "Réflexions sur l'effectivité du droit ", in Curapp (éd.), Les usages sociaux du droit, Paris, PUF, 1989, p. 140.

\section{Un encadrement juridique pernicieux}

Le cadre juridique de l'activité de transporteur routier de marchandises n'est pas aussi serré qu'il y paraît à première vue. Ce relâchement peut se mesurer à l'aune d'un corpus normatif dont les éléments constitutifs seraient l'existence de règles juridiques impératives couvrant l'ensemble des dimensions de l'activité, respectant la hiérarchie des sources du droit et s'intégrant aisément dans un système juridique plus vaste.

Il y a en particulier une évolution proprement juridique puisqu'il existe un mouvement complexe de " délégalisation». Sous ce vocable, nous voulons désigner une "certaine réduction de la pression juridique ", sans que cela se traduise principalement par une réduction quantitative des règles applicables. Il y aurait plutôt comme un relâchement de la cohérence du corpus de règles applicables au secteur du transport routier de marchandises. Pour s'exprimer de façon plus traditionnelle et imagée, nous pourrions dire que ces règles connaissent un moindre "rayonnement ", une "dévitalisation ", pour indiquer le mouvement général selon lequel, bien que toujours plus nombreuses, elles ne s'appliquent individuellement qu'en des circonstances plus limitées et de façon plus distanciée. Certains auteurs lient d'ailleurs l'inflation juridique au phénomène de la délégalisation " en raison de la dévaluation des lois qui en résulte immanquablement " $?$.

Cette réduction de la pression juridique a pour raison une "auto-neutralisation du droit " 8 ou des évolutions législatives partielles venant fragiliser l'application d'autres règles non modifiées. Un mouvement de délégalisation peut effectivement être associé à l'existence d'une réglementation touffue.

On constate d'abord qu'un mouvement général de libéralisation est venu réduire la place de la réglementation impérative applicable au secteur des transports routiers de marchandises (I.1). Ce mouvement de libéralisation s'est accompagné de la mise en place entre établissements d'une organisation de l'exploitation en flux tendus. Ce nouveau mode d'exploitation, qui vise à réduire au minimum les stocks des entreprises, a entraîné une intensification du travail des conducteurs routiers ${ }^{9}$. Cette tendance semble avoir fragilisé l'application effective des règles d'ordre public maintenues.

On remarque ensuite que les spécificités de la réglementation ne facilitent pas non plus sa bonne application (I.2). Les singularités de cette réglementation sont comme une invitation à ne pas la respecter. Il y a donc comme une certaine réduction de la coercition entretenue par le droit. Le droit ne trouve plus à s'appliquer avec la même force à l'ensemble des situations que rencontre un transporteur ${ }^{10}$. 


\section{I.1. Un contexte général de libéralisation}

Dès 1934, le secteur des transports routiers de marchandises a été fortement réglementé par une législation visant à la coordination entre les modes de transport ${ }^{11}$. Pour limiter la concurrence faite par la route au chemin de fer, l'accès à la profession et surtout l'exercice de celle-ci ont été encadrés. Deux de ces principaux mécanismes doivent être rappelés : premièrement, le droit pour une entreprise de transport public d'exploiter un véhicule d'un certain tonnage était soumis à autorisation et le nombre de licences délivrées par l'administration était contingenté. En second lieu, il n'y avait pas de liberté des prix concernant les contrats de transport. Or, ces dispositions ont été largement assouplies au cours des années 1980. Parallèlement, une " politique globale des transports " a été définie par l'importante loi d'orientation des transports intérieurs du 30 décembre 1982 (la LOTI).

En vertu de cette loi-cadre, le décret n n $^{\circ} 6-567$ du 14 mars 1986 disposait que les transports effectués en "zone courte» étaient autorisés sans limite dès lors qu'il y avait une inscription au registre des transporteurs. Pour les transports qui dépassaient donc le cadre d'une zone courte, le principe était celui d'une autorisation attribuée pour une durée illimitée par l'administration, mais sans contingentement précis. La tarification obligatoire existante a par ailleurs été abrogée par le décret n 88-638 du 6 mai 1988.

La déréglementation en matière de transports a donc principalement consisté à substituer un ensemble de règles impératives à d'autres règles autorisant une plus grande souplesse d'exploitation. Cette tendance n'est d'ailleurs pas propre au droit des transports et se retrouve notamment en matière de droit du travail 12 .

Ces réformes ont eu un impact important sur la profession et plus particulièrement sur les conditions de travail des chauffeurs routiers. Une véritable dérégulation des relations du travail s'est engagée, que l'on ne peut saisir sans rappeler brièvement combien le secteur des transports routiers est profondément hétérogène. La rivalité entre de nombreuses entreprises s'y double d'une concurrence entre les chauffeurs eux-mêmes, sur un marché du travail largement ouvert.

En premier lieu, l'ensemble des transports de marchandises par route n'est pas le fait des seuls transporteurs publics. Une proportion non négligeable de ces transports est assurée, en compte propre, par les entreprises du secteur industriel notamment ${ }^{13}$. Les chauffeurs routiers de ces entreprises ne sont pas concernés par la réglementation spécifique du secteur des transports publics (en particulier, le décret n $83-40$ du 26 janvier 1983 et l'accord " grands routiers" du 23 novembre 1994). Ils sont par
11. Décret du 19 avril 1934 , modifié par un décret du 12 novembre 1938.

12. A. JeAMMAUD et A. LYoNCAEN, Droit du travail, démocratie et crise, Arles, Actes Sud, 1986 , p. 39 ; A. SUPIOT, "Déréglementation des relations de travail et autoréglementation de l'entreprise ", Droit social, 3, 1989 ; M. BONNECHERE, "Les tendances à la déréglementation et leur incidence sur la théorie des sources en droit du travail », Droit social, 1 , 1990 ; S. SIMITIS, « Le droit du travail a-t-il encore un avenir ? ", Droit social, 7/8, 1997, p. 655.

13. P. MERLIN, Les transports en France, Paris, La Documentation française, 1994, p. 56 ;

M. BERNARDET, Le transport routier de marchandises, Paris, Economica 1997, p. 6 et suiv. 


\section{S. Carré}

L'état du droit dans le transport routier de marchandises : une réglementation en trompe-l'œil
14. MINISTËRE DE L'ÉQUIPEMENT

DAEI-SES, L'utilisation des véhicules de transport routier de marchandises en 1995, septembre 1996.

15. Cf. le Contrat de progrès, 1994, p. 2 ; cf. encore B. LEFEBVRE, 1996, art. cité, p. $82 ;$ F. OCQUETEAU et J.-C. THOENIG, « Mouvements sociaux et action publique : le transport routier de marchandises ", Sociologie du travail, 4, 1997, p. 404, 416. contre assujettis à la réglementation communautaire sur les temps de conduite. Ils bénéficient encore de la réglementation de droit commun sur la durée du travail ainsi que des avantages conventionnels propres à leur secteur d'activité.

Mais une entreprise du secteur industriel ou de la distribution peut trouver plus avantageux de sous-traiter les opérations de transports. Elle a alors recours à un transporteur routier. Si celuici emploie un chauffeur salarié, il va devoir respecter les règles sociales propres à ce secteur. Mais il est également possible de recourir à un artisan chauffeur. La seule réglementation applicable à ce dernier devient ce faisant la réglementation communautaire sur les temps de conduite.

Aussi la libéralisation intervenue dans les transports routiers de marchandises a-t elle modifié l'équilibre des relations du travail dans ce secteur en favorisant certains de ses acteurs aux dépens d'autres. De cette façon, le transport en compte propre a régressé au profit des transporteurs publics. Toutes distances confondues, le trafic routier de marchandises en compte propre est passé de 27,6 milliards de tonnes/kilomètre en 1981 à 30,5 en 1995. Dans le même temps, toutes distances confondues, le trafic routier de marchandises en compte d'autrui est passé de 61 milliards de tonnes/kilomètre à 101,5 ${ }^{14}$. Parallèlement, le transport public de marchandises a connu des transformations accélérées avec l'arrivée massive de petites entreprises, dont un grand nombre d'artisans.

En bref, pour cette activité de main-d'œuvre, la concurrence accrue a momentanément favorisé les entreprises capables de proposer la force de travail la moins coûteuse, celles à qui le droit commun sur la durée normale du travail n'était pas applicable, celles qui ne sont pas assujetties au droit du travail (les chauffeurs routiers artisans). Elle a favorisé l'irrespect de la réglementation $\mathrm{du}$ travail pour toutes les entreprises qui, devant appliquer cette législation à leurs conducteurs routiers salariés, ne pouvaient le faire sans perdre de l'argent ou des parts de marché. Ces entreprises, principalement de petite et moyenne taille, ont effectivement été confrontées à la concurrence des artisans et à la position dominante des chargeurs et des intermédiaires du transport, qui ont su jouer de l'éclatement de la profession ${ }^{15}$.

Enfin, la dérégulation des relations du travail, dont on perçoit bien qu'elle est avant tout une modification des "règles du jeu ", en favorisant certains statuts et en entraînant une application différente du droit, a été facilitée par le mouvement général de flexibilisation de la main-d'œuvre salariée. Les réformes intervenues dans les années quatre-vingt, visant à promouvoir les contrats de travail précaires et les accords dérogatoires en matière de durée et d'aménagement du temps de travail ont évidemment permis aux entreprises de s'adapter plus aisément à la rapide évolution du marché. Dans cette optique, l'accord « grands routiers » du 23 no- 
vembre 1994, par ses dispositions tout à fait particulières, peut être perçu comme l'aboutissement, au niveau conventionnel, de ce mouvement de flexibilisation (cf. infra).

Mais l'essentiel est, semble-t-il, ailleurs. Effectivement, la flexibilité accrue de la main-d'œuvre a participé au mouvement de " délégalisation " en rendant inopérantes les règles impératives subsistantes et en facilitant l'émergence de pratiques d'entreprises relevant de "l'infra-droit " ${ }^{16}$. Bien que la situation ne soit pas irréversible, comme le montrent les conflits sociaux de ces dernières années, la précarité de ces travailleurs empêche, en pratique, qu'ils obtiennent l'étroite application de ces règles.

Comment faire appliquer la minutieuse législation communautaire sur les temps de conduite lorsque le chauffeur, travailleur indépendant, n'est qu'un tractionnaire au service d'un seul donneur d'ordre, un transporteur, qui ne sous-traite son activité que pour répondre à l'augmentation ponctuelle de son volume d'affaires? De façon similaire, il devient difficile au conducteur sous contrat à durée déterminée, ou se trouvant en concurrence sur le marché de l'emploi avec de nombreux autres travailleurs de même qualification, de refuser le principe d'un salaire au forfait, dont il sait pourtant qu'il sera l'occasion de dépassements non rémunérés de la durée du travail ${ }^{17}$. Enfin, l'accord du 23 novembre 1994, en fixant des durées maximales de service supérieures à la durée maximale du travail autorisée par la loi, ne peut qu'inciter les employeurs à se référer à cette convention plutôt qu'au Code du travail.

\subsection{Une réglementation incohérente}

L'inobservation des règles juridiques applicables est facilitée par la faible cohérence des textes généraux et spécifiques qui régissent une matière. Les difficultés techniques de mise en œuvre, le flou qui s'instaure autour de certaines notions favorisent une application édulcorée de ces dispositions.

Finalement, pour un système juridique dont la pertinence tient à la cohérence des normes qu'il pose, comme la présence de notions juridiques compréhensives et récurrentes ou la conformité à la hiérarchie des sources de droit, les scories d'une législation sont comme un démenti à la force obligatoire des règles. Il n'est pas inutile de rappeler ici la thèse de Max Weber selon laquelle la légitimité du droit moderne est à relier au principe de légalité et plus précisément à la rationalité matérielle, sinon formelle des règles édictées ${ }^{18}$. À l'image d'un habit mal ajusté, le décret du 26 janvier 1983 relatif à la durée du travail dans les transports routiers est l'illustration d'un droit toujours mal adapté : l'employeur le prendra-t-il au sérieux ${ }^{19}$ ?

16. Infra-droit : « Tout ce qui, n'étant pas droit au sens strict (droit étatique ou régulation reconnue comme droit par l'État), participe néanmoins du phénomène juridique lato sensu " (A.J. ARNAUD [sous la dir.], Dictionnaire encyclopédique de théorie et de sociologie du droit, op. cit.).

17. M. HAUTDIDIER et

P. RAMACKERS, « La durée du travail est-elle encore contrôlable? Réflexions sur une évolution à partir de l'exemple des transports routiers », Droit social, 4, 1995 ; A. SUPIOT, " Temps de travail : pour une concordance des temps ", Droit social, 12, 1995, p. 953.

18. M. WEBER, Sociologie du droit, Paris, PUF, 1986, les chapitres VI et suiv.

19. Une délégitimation du droit du travail du fait de sa complexité croissante pourrait exister au sein même de l'Inspection du travail (P. AUVERGNON, « Contrôle étatique, effectivité et ineffectivité du droit du travail ", Droit social, 6, 1996, p. 607). 
S. Carré

L'état du droit dans le transport routier de marchandises: une réglementation en trompe-l'œil
20. Cette étude portant sur l'état du droit jusqu'au milieu des années 1990, nous ne tenons pas compte des réformes récentes. Ainsi celle entraînant la modification de la notion de " travail effectif " (nouvel article L. 212-4 Code trav.). De même, le décret du 26 janvier 1983 a été modifié par un décret du 27 janvier 2000.

21. Décret du 26 janvier 1983, modifié par les décrets du 3 août 1992 et du 26 février 1993, article $5 \S 3$.

22. R. DHOQUOIS et A. JEAMMAUD, Le droit capitaliste du travail, Grenoble, PUG, 1980, p. 63.
Alors que la loi fixe la durée hebdomadaire normale ou maximale de travail en fonction du temps de travail effectif réalisé par le salarié (L. 212-1, L. 212-4, L. 212-7 C. trav.), le décret de 1983 pose une autre notion, celle de temps de service, et donne sa propre définition du temps de travail effectif (article $5 \$ 1$ ) ${ }^{20}$. Ces distinctions ont permis d'inclure dans le calcul des temps de service les périodes pendant lesquelles certains chauffeurs routiers sont à la disposition de l'employeur tout en n'exerçant aucune activité particulière, comme lors d'attentes avant un déchargement.

Mais les différentes notions du temps de travail effectif restent malgré tout très proches tandis que le décret du 26 janvier 1983 ne donne aucune définition explicite des temps de service, ce qui rend ces distinctions peu lisibles. En particulier, l'une des dispositions les plus originales du texte de 1983, celle concernant les temps de mise à disposition, n'apparait pas dans la définition du temps de travail effectif, au sens du décret, tout en justifiant manifestement la différence d'intitulé entre temps de travail et temps de service.

Ces difficultés notionnelles se cumulent avec des règles particulièrement complexes à mettre en œuvre. Ainsi, le calcul du temps de travail effectif au sein des périodes de mise à disposition s'est longtemps basé sur un décompte particulièrement minutieux: seul un certain pourcentage du temps réellement écoulé était comptabilisé comme du temps de travail. À cela s'ajoutait la règle du "butoir ", entraînant une dissociation entre le calcul du temps de travail au sein des périodes de mise à disposition, au titre des dispositions sur la durée du travail, et ce même calcul, au titre de la rémunération du chauffeur ${ }^{21}$.

$\mathrm{Au}$ caractère alambiqué des dispositions du décret de janvier 1983, il faut adjoindre celles, non moins embrouillées, propres à la législation européenne. Ainsi, le règlement communautaire $n^{\circ} 3820 / 85$ impose à tout conducteur routier de s'arrêter de conduire durant au moins quarante-cinq minutes après quatre heures trente de route. Cette coupure est fractionnable sous forme de pauses d'au moins un quart d'heure. Par ailleurs, le même règlement dispose qu'une période de repos doit être égale à une heure au moins, le chauffeur étant alors totalement libre de son temps. Mais si le conducteur s'arrête de conduire moins d'un quart d'heure, sans être pendant ce temps à la disposition de son employeur, il ne s'agit ni d'une coupure ni d'un repos...

Il est clair au travers de ces exemples que la transformation d'une législation en une réglementation pointilleuse rend d'autant moins probable une stricte application, alors même que cette réglementation tend à renforcer la contrainte par le détail de ses dispositions. En d'autres termes, plus il existe d'obligations précises, plus il y a "mécaniquement " d'opportunités à transgresser ces règles 22 . 
Bien entendu, cette réglementation pointilleuse est d'autant Droit et Société 46-2000 plus impuissante à réguler les conduites que les personnes à qui elle s'adresse ont d'importantes marges d'autonomie dans l'organisation matérielle de leur activité. Le constat a d'ailleurs été fait de l'incapacité croissante des systèmes de production centralisée de normes à encadrer les pratiques, du fait de " la complexité des rapports sociaux nés de la modification des systèmes productifs à la suite de la crise du mode taylorien de production " 23 .

Enfin, il se peut que le destinataire de ces textes soit peu disposé à les accepter, parce que leur difficile combinaison semble indiquer des fissures dans l'édifice juridique, la légalité de certaines dispositions réglementaires pouvant d'ailleurs être mise en doute. De la sorte, le décret de janvier 1983 utilise la notion de "temps de service ", que ne connaît pas la loi. Or, celle-ci a seule compétence pour fixer les "principes fondamentaux " en droit du travail (article 34).

Le respect par l'employeur de la durée maximale des temps de service fixée par le décret aboutit à dépasser la durée maximale du travail (48 heures par semaine) instituée par la loi. Mais cette situation n'intervenait que lorsque les " temps à disposition de l'employeur " étaient particulièrement réduits au regard des périodes de pleine activité, puisque seul un certain pourcentage de ces temps étaient décomptés en temps de travail effectif. Depuis la réforme du 12 décembre 1996, les temps à disposition étant intégralement comptés dans un temps de travail effectif, la durée maximale des temps de service indiquée au décret apparait donc toujours supérieure à la durée maximale du travail sur la semaine.

La faible cohérence de l'ensemble de ces textes éclate au grand jour quand des dispositions en viennent à être annulées par le Conseil d'État ${ }^{24}$. Mais un tel événement masque un phénomène plus important: la rareté même des recours en annulation ou l'invocation d'une exception d'illégalité envers certaines dispositions juridiques applicables à ce secteur d'activité. Tout a lieu comme si le contenu de ces textes et la manière dont ils étaient en pratique abordés rendaient un recours en justice inadapté ou malvenu.

D'ailleurs, les partenaires sociaux ont, en quelque sorte, consacré le caractère pernicieux de la réglementation étatique en concluant une convention collective si dérogatoire à la loi que le caractère illicite de certaines de ses dispositions ne fait aucun doute ${ }^{25}$. Ce volontarisme des partenaires sociaux, qui fait fi de dispositions légales impératives, peut d'ailleurs apparaitre comme une excellente illustration d'un pluralisme juridique 26 .

Certes, des conventions collectives dérogatoires à la loi sont aujourd'hui possibles, sur la base des articles L. 132-26 et L. 212-2 du Code du travail. Cependant, la validité de tels accords est sou-

23. J.-P. BONAFE-SCHMITT, « Pour une approche socio-juridique de la production des normes dans les relations de travail ", Droit et Société, 27, 1994, p. 343.

24 . De la sorte, l'article $l^{\text {er }} d u$ décret n' 96-1115 du 19 décembre 1996 a été jugé illégal par cette juridiction (CE 5 octobre 1998 BTL n` 277012 octobre 1998, p. 723), sur recours de l'UNOSTRA.

25. M. HAUTDIDIER et

P. RAMACKERS, 1995, art. cité, p. 335 et suiv. Illégalité confirmée par un récent arrêt de la Cour de cassation (Crim. 24 novembre $1998 \mathrm{BTL}^{\circ} 2783,18$ janvier 1999).

26. S. CARRÉ, « Un exemple de pluralisme juridique : l'accord Grands Routiers du 23 novembre 1994 ", Revue juridique de l'Ouest, 3, 1999. 
S. Carré

L'état du droit dans le transport routier de marchandises : une réglementation en trompe-l'œil mise à certaines conditions de fond. La convention collective ne peut déroger à tout. Selon l'article L. 212-2 alinéa 3 du Code du travail, une convention collective étendue peut seulement déroger aux décrets d'application concernant l'aménagement de la durée légale du travail pour leurs dispositions relatives à "l'aménagement et à la répartition des horaires de travail à l'intérieur de la semaine, aux périodes de repos, aux conditions de recours aux astreintes, ainsi qu'aux modalités de récupération des heures de travail perdues».

Or, l'accord du 23 novembre 1994 sur «le temps de service, les repos récupérateurs et la rémunération des personnels de conduite marchandises grands routiers ou longue distance " stipule notamment que, à compter du 1 1er octobre 1995 et jusqu'au 31 décembre 1996, puis à partir du $1^{\text {er }}$ janvier 1997, les temps de service ne pourront être supérieurs, respectivement, à 60 heures et 56 heures par semaine (article VII.1 de l'accord). Ces durées limites sont évidemment supérieures à celles prévues par le décret $n^{\circ} 83$ 40 du 26 janvier 1983 qui fixe, nous l'avons vu, la durée maximale de service à 52 heures sur une semaine (article $5 \S 4$ ).

Ces dispositions conventionnelles dérogatoires aux décrets d'application concernant l'aménagement du temps de travail sont donc illégales en ce que la loi n'a jamais autorisé par voie conventionnelle un dépassement de la durée maximale du travail ou de service.

Par ailleurs, l'accord de novembre 1994 ćtablit un mode de calcul de la durée du travail sur le mois et non sur la semaine (article IV.2). Or, les accords dérogatoires ne doivent pas moins maintenir un mode de calcul sur la semaine. En cas d'annualisation du temps de travail ou d'accord de cycle, c'est la durée moycune de travail sur une semaine qui doit rester en dernière analyse le point de référence. Cette disposition semble donc être contraire tant à l'article L. 212-2 du Code du travail qu'à l'article L. 212-5.

$\mathrm{Au}$ surplus, il aurait été nécessaire que ces dispositions fassent l'objet d'un arrêté d'extension pour être valides puisque seule une convention collective étendue peut comporter des dispositions dérogatoires à certaines questions touchant à l'aménagement du temps de travail et des horaires (L. 212-2 C. trav.). Or, il n'a jamais été procédé à la procédure d'extension.

Sans aller plus loin dans la démonstration, il apparaît que nous sommes en face d'une législation qui, tout à la fois, tente de répondre aux attentes et aux pratiques des transporteurs routiers par une réglementation largement dérogatoire et cherche à les encadrer par des dispositions juridiques minutieuses. Il en résulte une législation inextricable. Le phénomène de la déjuridicisation se présente donc comme un délitement de la cohérence interne d'un corps de règles, une altération de catégories juridiques, sous 


\section{Un encadrement juridique permissif}

La législation existante est restée largement ineffective. Ce phénomène préexistait au mouvement de déjuridicisation enclenché à partir des années quatre-vingts. Traditionnellement, ce milieu professionnel n'est donc pas enclin au respect scrupuleux des règles juridiques qui lui sont applicables. Cette latitude prise par rapport à la loi n'apparaît pas sans conséquence sur la consistance du droit lui-même. C'est en réalité l'existence même des règles d'ordre public qui est contestée du fait de cette ineffectivité. Des règles impératives gardent-elles un caractère de droit positif lorsqu'elles deviennent largement inopérantes? On peut le contester. Il est du moins possible de dire que leur contenu ne permet pas de décrire, à lui seul, la "réalité normative " 28 . Toute la question étant alors de préciser la consistance et les limites du droit existant.

Une solution est d'affirmer que nous sommes en face d'un phénomène de non-droit (II.1). On ne peut cependant s'en tenir à cette constatation. En effet, y a-t-il seulement un recul du droit ? Il semble que derrière ce recul se profile un autre phénomène : nous serions en face d'un ordre public de façade, les autorités publiques permettant dans une certaine mesure sa transgression. Ces règles, ni véritablement impératives, ni supplétives, pourraient être qualifiées de permissives (II.2).

\section{II.1. Les insuffisances d'un droit impératif}

Jean Carbonnier caractérise notamment une situation de nondroit par une résistance des faits au droit, par l'incapacité du droit à enserrer une réalité, à la réglementer, malgré son apparente présence. Il parle encore d'une démission du droit. Mais il y a, en définitive, de nombreux phénomènes distincts derrière cette idée 29 .

La résistance des faits au droit trouve d'abord ses racines dans les particularités sociologiques du milieu professionnel concerné. Il n'est pas dans notre propos d'analyser ce point. Il nous suffira de constater qu'il existe depuis longtemps, et quelle que soit l'évolution de la législation, un important écart à la loi.

Par ailleurs, ainsi que nous l'avons souligné, la complexité et les incohérences de cette législation ne sont, semble-t-il, que le reflet des difficultés à encadrer les pratiques professionnelles. Mais il n'y a pas là une démission du droit. Il y a plutôt un échec.

Par contre, si l'on s'en tient à une conception restrictive du droit fondée sur la prééminence de règles écrites et obligatoires, il y a comme une véritable "démission " quand la réglementation et
27. L. ASSIER-ANDRIEU, Le droit dans les sociétés humaines, Paris, Nathan, 1996, p. 55-56.

28. A. SUPIOT, « Recherche sur l'application des textes relatifs à la pollution de l'eau d'origine industrielle ", La Semaine juridique, 13, 1975, I doctrine 2692 ; P. LASCOUMES et J.-P. LF BOURHIS, « Des "passedroits" aux passes du droit. La mise en œuvre socio-juridique de l'action publique ", Droit et Société, 32, 1996, p. 64, 69.

29. J. CARBONNIER donne à la notion de non-droit une acception très large, dont la résistance des faits au droit n'est qu'une modalité (Flexible droit, Paris LGDJ, 5 éd., 1983, p. 25 et suiv.). 


\section{S. Carré}

L'état du droit dans le transport routier de marchandises : une réglementation en trompe-l'œil
30. Institut national de recherche sur les transports et leur sécurité.

31. Rapport Dobias, janvier 1993, p. 7 et 11, et annexe 3, p. 4.

32. En 1991, le taux de dépassement de la vitesse-limite était globalement de $42 \%$ (Rapport Dobias, janvier 1993, annexes 3 et 8 ).

33. Les contrôles sur route doivent seulement porter sur la bonne application du règlement européen n`3820 du 20 décem. bre 1985 (circulaire $n^{\circ} 86-66 \mathrm{du}$ 29 septembre 1986 ).

34. B. LEFEBVRE, « Usages des réglementations dans le transport routier : approche ethnologique en région Rhône-Alpes ", in Transports 93, professions en devenir, op. cit., p. 167. la pratique administrative favorisent directement l'inapplication des règles. Le droit semble battre en retraite, effectuer une opération de repli, laissant à l'abandon des secteurs que le législateur avait entendu réguler.

\section{La résistance des faits au droit}

Selon le rapport Dobias, rapportant une enquête de l'LNRETS ${ }^{30}$ : " En 1980, 60\% des conducteurs travaillaient plus de 60 heures par semaine et $53 \%$ des entreprises contrôlées en 1979 étaient en infraction grave et chronique contre $22 \%$ qui respectaient strictement les textcs. » L'inapplication du droit s'est maintenue dans les années quatre-vingts, les mêmes causes ayant les mêmes effets, et la réglementation nouvelle étant plus le reflet de l'impuissance du droit à encadrer une pratique rétive qu'une démarche visant à fixer de façon claire et simple ce qui peut être permis ou interdit.

De cette façon, en 1982/1983, une deuxième enquête de l'INRETS constatait que la durée du travail hebdomadaire des chauffeurs routiers salariés atteignait 59 heures par semaine, soit nettement plus que la durée maximale du travail fixée par la législation. Enfin, l'enquête INRETS réactualisée au début des années 1990 ne montre aucune évolution notable des temps de travail. En 1991, $81,8 \%$ des infractions à la réglementation européenne relevées sur route (et $86,1 \%$ de ces infractions relevées en entreprise) concernaient les temps de conduite et de repos des conducteurs routiers ${ }^{31}$.

Une autre enquête, effectuée par le ministère de l'Équipement, indiquait que plus de la moitié des poids lourds enfreignaient la vitesse limite sur autoroute ${ }^{32}$. Le contrôle des excès de vitesse est par ailleurs rendu difficile du fait de l'équipement de nombreux camions en matériel de télécommunication (du type " $C B$ ») et de la défense de faire a posteriori la constatation de l'infraction grâce au chronotachygraphe ${ }^{33}$.

En réalité, le milieu des transporteurs routiers et celui des conducteurs, du moins celui des PME et des artisans, ont en commun une culture commune qui les éloigne d'un comportement légaliste. "Les relations entre ouvriers, employés, cadres, entrepreneurs sont l'objet d'un consensus selon lequel le recours au droit n'est la tradition ni de la maison, ni de la profession ${ }^{34}$. »

De l'irrespect de ces règles, alors qu'elles sont impératives et fixent des normes précises, on pourrait affirmer qu'elles sortent de l'ordonnancement juridique. Elles ne seraient plus de droit positif. L'idée est séduisante mais aisément critiquable: à moins d'une inapplication totale ou d'une application faite seulement par complète inadvertance, la règle a manifestement un impact. Il n'est d'ailleurs pas nécessaire qu'une personne connaisse une règle ou en ait conscience pour que cette disposition ait un effet. Il 
suffit que cette personne suive les usages de son milieu, s'ils sont

influencés par ladite règle 35 .

Mais cette réglementation n'est-elle justement pas suivie par inadvertance ? Il est vrai que la situation ressemble fort à celle-ci. Bruno Lefebvre souligne que le droit n'est suivi que dans la mesure où il rencontre les pratiques en vigueur pour gérer le personnel ${ }^{36}$. De même Pierre Hamelin indique que, "concernant toutes les réglementations liées à la circulation routière et à sa sécurité, la norme demeure le suivi des règles au gré des aléas de la réalisation de la production quotidienne $" 37$.

On constate cependant qu'il existe tout de même un usage de cette réglementation par la profession. Flle ne la méconnaît pas mais n'envisage de la respecter que si elle la sert dans son exploitation ou ne la contrarie pas. Par ailleurs, les entreprises de transport rattachées à un grand groupe ont une attitude nettement plus légaliste que les petites entreprises indépendantes. Aussi l'hypothèse du non-droit nous semble-t-elle mal adaptée, sinon pour indiquer qu'il existe effectivement un décalage particulièrement important entre la lettre de la loi et son application. Mais cette hypothèse ne nous permet pas de qualifier précisément les circonstances présentes, où le droit est là comme sous une autre forme que celle qui se laisse apparemment voir, celle d'un tissu réglementaire pointilleux.

\section{Le repli du droit}

Si la réglementation existante n'a jamais été réellement appliquée, c'est aussi que son irrespect n'a jamais été véritablement sanctionné. Face à un milieu professionnel réfractaire, il aurait donc fallu soit prendre les moyens de son application, soit changer radicalement le contenu et les caractéristiques des normes contenues dans ces textes. La seconde solution a certes été envisagée puisque des dispositions tout à fait spécifiques ont été promulguées. Cependant, l'impossibilité de heurter frontalement les règles d'ordre public inscrites au Code du travail a finalement conduit à une autre voie : celle consistant à organiser, jusqu'à un certain point, l'inapplication de ces règles.

De cette façon, l'article 9 de la LOTI dispose: "Est nulle de plein droit, dans les contrats de transport et dans les contrats de travail, toute clause de rémunération principale ou accessoire de nature à compromettre la sécurité, notamment par l'incitation directe ou indirecte au dépassement de la durée du travail et des temps de conduite autorisés. " De ce point de vue, la rémunération au forfait, largement pratiquée dans la profession, sans décompte précis des temps de travail n'est-elle pas une incitation indirecte au dépassement des heures de travail et de conduite autorisées?
35. C. MINCKE, 1998, art. cité, p. 119.

36. B. LeFEBVRE, 1992, art. cité, p. 174.

37. P. Hamelin, 1992, art. cité, p. 109. 


\section{S. Carré}

L'état du droit dans le transport routier de marchandises : une réglementation en trompe-l'œil

38. Il a fallu attendre ces dernières années pour que des tribunaux soit saisis de la question et aillent en ce sens (Cour d'appel Paris, $1^{\text {er }}$ ch., 1er avril 1997 BTL 1997, p. 311).

39. De plus, les commissions des sanctions administratives ayant compétence pour rendre un avis sur les sanctions que pourront prendre les préfets ne se sont pas réunies durant plusieurs années. Elles ne vont être activées qu'à partir de 1993 (BTL n' 2626 du 11 septembre 1995, p. 582 ; BTL n` 2704 du 28 avril 1997, p. 315).

40. Ainsi, la surcharge d'un cdmion est simplement punie d'une amende forfaitaire de $900 \mathrm{~F}$ (surcharge par rapport au poids total autorisé par la carte grise, $R 238$ C. de la route). 41. Plusieurs réformes sont alors intervenues : décret $n^{\circ} 92$ 699 du 23 juillet 1992 ; loi n’ 95 96 du ler février 1995 ; décret n' $95-602$ du 5 mai 1995 ; loi n' $98-69$ du 6 février 1998.

42. Il semble néanmoins que les contrôles aient été plus nombreux et précis à partir de 1994 (circulaire interministérielle de mars 1994 ; note de la direction des transports terrestres du 14 avril 1995 : BTL n' 2626 du 11 septembre 1995, p. 581 et suiv. ; BTL n' 2723 du 13 octobre 1997, p. 688).

43. F. OcQueteau et

J.C. THOENIG, 1997, art. cité, p. $397,407$.

44. Rapport Dobias, annexe 8, p. 2.
Cependant, ni les intéressés, ni l'administration n'ont tenté de contester ouvertement et officiellement cette pratique ${ }^{38}$.

De même, l'article 32 de la LOTI dispose que les contrats de transport doivent prévoir, "à peine de nullité, l'estimation des temps nécessaires à l'exécution des différentes tâches ». Cependant, à notre connaissance, cette sanction n'a jamais été mise en œuvre. Le législateur a d'ailleurs ressenti le besoin de mettre en place une autre sanction. Le non-respect de certaines dispositions fixées par la LOTI (dont l'article 32) a notamment pour sanction le retrait des autorisations de transport délivrées par l'administration (article 37). Mais l'application de cette sanction ne peut intervenir qu'en cas de " manquements graves ou répétés ». Un certain écart à l'application du droit est donc explicitement fixé par la loi elle-même ${ }^{39}$. En 'éalité, il n'a été parfois prévu de sanctions, ou les sanctions existantes n'ont été renforcées, qu'à partir de 1992 , soit de nombreuses années après la promulgation des lois et règlements régissant la profession.

Concernant les sanctions pénales existantes, la modicité de certaines amendes ajoutée au faible nombre des contrôles incitent les transporteurs à ne pas respecter la réglementation : il y a effectivement plus d'avantages économiques (augmentation de la productivité) à ne pas en tenir compte ${ }^{40}$. Les sanctions pénales encourues en cas de non-respect de la législation sociale sont principalement des contraventions de quatrième classe. Seule l'ordonnance $n^{\circ}$ 58-1310 du 23 décembre 1958 énonce quelques délits relatifs aux opérations de contrôle.

De nombreuses pratiques incitant ou visant à enfreindre la réglementation ne sont pas, à l'époque, pénalement sanctionnées. De cette façon, le fait pour un employeur ou un donneur d'ordre d'inciter au non-respect de la législation routière ou de la réglementation relative à la durée du travail ne reçoit aucune sanction.

Le législateur n'a véritablement commencé à durcir les sanctions pénales qu'à compter du grave conflit social du mois de juin $1992^{41}$. De plus, les contrôles sont toujours restés rares ${ }^{42}$, la profession ayant même obtenu de l'administration qu'ils soient diminués, notamment sur route ${ }^{43}$. La directive européenne $n^{\circ}$ 88-599 du 23 novembre 1988 a certes défini ce que devait être le contrôle minimal engagé par les États pour l'application des règlements européens du 20 décembre 1985. Il a été fixé que le contrôle, tant sur route qu'en entreprise, devait permettre de contrôler au moins $1 \%$ des jours de travail des conducteurs de véhicules (article 2-2). Cet objectif pourtant réduit n'était toujours pas atteint en 199244.

La liste des fonctionnaires habilités à contrôler la réglementation applicable aux transporteurs est néanmoins impressionnante. Mais beaucoup d'entre eux sont en partie distraits de cette fonction par d'autres tâches (ex. : la gendarmerie, les CRS). Les fonc- 
travail sont en fait peu nombreux. En effet, en pratique, le respect de la législation sur la durée du travail n'est vérifié que par les inspecteurs et contrôleurs du travail " transport ». La gendarmerie, la police et les contrôleurs des transports dépendant du ministère de l'Équipement contrôlent plus spécifiquement l'application de la réglementation européenne sur les temps de conduite et de repos, des règles concernant la coordination des transports et de celles relatives au Code de la route.

Or, on ne comptait, en 1995, que 138 inspecteurs du travail et de la main-d'œuvre des transports. En 1993, il y avait 289 contrôleurs des transports terrestres, leur nombre n'ayant quasiment pas varié depuis 1989 45.

De plus, seule une faible proportion des infractions constatées a fait l'objet de procès-verbaux ${ }^{46}$. De fait, une circulaire ministérielle en date du $1^{\text {er }}$ juin 1990 préconisait «d'attacher toute leur importance aux déclarations de l'employeur permettant de dégager sa responsabilité et d'incriminer un tiers, dès lors qu'elles sont assorties de justifications recevables ", quand il était constaté que l'employeur avait incité son chauffeur à enfreindre directement ou non la législation sur la durée du travail et les temps de conduite (article 9 de la LOTI). Par ailleurs, plus de $80 \%$ des amendes prononcées étaient égales ou inférieures au minimum prévu pour les contraventions de quatrième classe ${ }^{47}$.

On peut donc parler d'une "démission " du droit par référence à ce que prétend être la législation applicable au secteur, c'est-à-dire une législation impérative. Mais, bien entendu, le terme est inadéquat dès lors que l'on constate que les dysfonctionnements, les antinomies ou les faiblesses rédactionnelles forment en réalité un tout, qu'il y a finalement une cohérence derrière l'apparent désordre!

\section{II.2. L'hypothèse d'un droit indicatif}

L'État a mis en place tout un dispositif visant à permettre un écart significatif aux normes législatives et réglementaires. Jusqu'au début des années 1990, ce dispositif est pour une part inscrit directement dans les textes. Il existe aussi en creux par l'absence ou l'insignifiance des sanctions concomitantes. Il apparait enfin au travers de la consistance des contrôles administratifs et des sanctions effectivement prises à l'encontre de ceux qui ne respectent pas la réglementation apparemment imposée ${ }^{48}$.

L'attitude compréhensive des pouvoirs publics est ancienne et antérieure aux années quatre-vingts. L'État a régulièrement assoupli les règles relatives au contingentement du trafic par voie routière, vidant de son contenu la législation sur la coordination des transports. Cette législation de même que la tarification routière

45. Rapport Salini, BTL n' 2626

11 septembre 1995, p. 581-582. 46. Par exemple, 1777 infractions à la réglementation sociale sur 24200 infractions relevées durant le premier trimestre 1994 ont fait l'objet d'un procès-verbal, soit 13,6\%. Les condamnations pénales pour non-respect du décret 83.40 étaient rarissimes (BTL $\mathrm{n}^{\circ} 2592$ du 12 décembre 1994, p. 883). 47. Soit $1300 \mathrm{~F}$ en 1991 (Rapport Dobias, janvier 1993, annexe 8, p. 3). Selon une enquête du CNT, $90 \%$ des amendes prononcées ont été inférieures au minimum légal, et $27 \%$ d'entre elles ne dépassaient pas $100 \mathrm{~F}$ en 1996 (BTL 1997, p. 560).

48. Mais ce phénomène n'est pas propre au secteur des transports routiers, cf. P. AUVERGNON, 1996, art. cité, p. 606 ;

É. SERVERIN, «L'application des sanctions pénales en droit so. cial : un traitement juridictionnel marginal ", Droit social, 7-8, 1994, p. 657. 


\section{S. Carré}

L'état du droit dans le transport routier de marchandises : une réglementation en trompe-l'œil

49. Rapport Dobias, p. 10.

50. P. HAMELIN, 1992, art. cité, p. 107 et 108.

51. A. Supiot, 1975, art. cité ; cf. également A.-J. HOEKEMA, " La production des normes juridiques par les administrations ", Droit et Société, 27, 1994, p. 318 et suiv. ; A.-J. ARNAUD, "Le droit comme produit ", Droit et Société, 27, 1994, p. 298. Sur la notion de "fait normatif ", cf. G. GURVITCH, "Problèmes de sociologie du droit ", in

G. GURVTrCH (sous la dir.), Traité de sociologie, tome II, Paris, PUF, 1968.

52. T. GEIGER fait clairement la différence entre les coutumes " sociologique " et " juridique " : l'une et l'autre fixeraient une règle obligatoire, mais seule la coutume juridique serait constatée par une tierce personne (sur T. Geiger, cf. H.E. ROHRER, La sociologie juridique de Théodore Geiger, Thèse, Paris, 1971). La doctrine (par exemple : F. GENY, Méthodes d'interprétation et sources en droit privé positif, Paris, 1899) constate généralement l'existence d'une coutume juridique, à partir de la régularité d'une pratique collective et du sentiment que celle-ci est obligatoire. La coutume juridique existerait alors par elle-même, indépendamment de sa consta. tation par le juge.

53. Ainsi, certaines normes permissives fixées par l'administration, qui n'auraient qu'une existence fonctionnelle (J.-M. FEVRIER, " Remarques sur la notion de norme permissive ", Recueil Dalloz, 28, 1998, chron., p. 271 et suiv.). obligatoire étaient définies et contrôlées en commun avec les représentants de la profession. Il en a résulté, bien avant la libéralisation de ce secteur, la fixation de prix bien inférieurs aux planchers fixés par la Tarification routière obligatoire 49 .

Il est donc possible d'affirmer que l'Etat a été «le principal agent de légalisation de la non-application d'un principe législatif ${ }^{50}$. Pour une situation approchante, celle de la politique de l'État en matière de pollution de l'eau d'origine industrielle, Alain Supiot pouvait similairement déclarer que "la règle effectivement appliquée est finalement la résultante de la règle énoncée, d'une part, et de la régularité à laquelle se soumet l'Administration dans son application, d'autre part $» 51$.

Que dire de l'état du droit dans ce secteur d'activité sinon qu'il est différent de celui qui se donne à voir ? Mais comment qualifier plus précisément ce phénomène? L'idée d'une zone de non-droit, nous l'avons vu, est insuffisante. Celle de l'existence d'un infradroit ne nous semble pas non plus totalement adéquate. Il ne s'agit pas précisément de l'apparition d'un phénomène juridique parallèle qui émanerait spontanément d'un groupe social. Il n'y a pas, en particulier, l'émergence d'usages ou de coutumes " contra legem".

Si coutumes il y a, il s'agit de coutumes au sens sociologique du terme, c'est-à-dire de pratiques communément suivies. Mais il n'y a pas de coutumes ni d'usages caractérisant un phénomène juridique. La notion d'obligation est absente. De plus, aucun organisme, aucune tierce personne ne se trouve réellement présent pour constater l'existence d'une règle coutumière et l'opposer à ceux qui ne l'auraient pas prise en compte ${ }^{52}$. En réalité, les pratiques de la profession ne paraissent pas guidées par un ensemble de normes positives qu'il serait du moins recommandable de suivre mais par une contrainte économique fluctuante : ne pas perdre un marché, faire des bénéfices, payer les créanciers, réduire les coûts de revient...

Il est par contre possible de soutenir que le législateur, ayant posé un certain nombre de règles, puis en en facilitant la transgression, établirait en quelque sorte une sphère de permissivité, au sens d'une zone de tolérance. Mais il ne s'agit pas véritablement de permissions. L'écart à la règle n'est pas le résultat d'une autorisation. Il ne prend pas la forme d'une exception explicite à une obligation ${ }^{53}$.

Les règles juridiques en vigueur auraient plutôt le rôle d'étalon. Elles permettraient à chacun de se situer juridiquement. En fonction de la position des acteurs par rapport à la réglementation, ces dispositions seraient susceptibles d'être utilisées ou invoquées lors une négociation commerciale ou à propos d'une transaction. Elles faciliteraient l'appréhension et la mesure du risque 
juridique encouru et permettraient, ce faisant, certains choix de Droit et Société 46-2000 gestion tel le recours à la sous-traitance. En cela, la règle de droit connaîtrait déjà une forme d'effectivité 54 .

La règle de droit devient une référence. Sa fonction serait plutôt symbolique, par l'indication faite à chacun des confins d'une situation juridiquement protégée. La marque de cette frontière n'est d'ailleurs pas nécessairement sans influence sur l'importance des comportements "déviants": de subtils calculs peuvent s'en suivre, comme de savoir jusqu'où l'on peut se permettre d'aller sans encourir le risque d'avoir un comportement trop voyant... Mais cette règle n'a plus pour fonction d'être étroitement appliquée. Elle n'est donc plus véritablement une disposition d'ordre public. Telle serait, en définitive, la consistance du droit en vigueur.

Nous rejoignons ici les auteurs qui soulignent que le droit n'a pas pour fonction première d'imposer des comportements particuliers aux personnes, mais qu'il est avant tout un modèle pour l'action ${ }^{55}$. Nous ajouterons à cela que, dans le cas présent, le corpus juridique se présente apparemment comme imposant ou interdisant une suite de comportements. Mais ce corpus comporte par ailleurs des caractéristiques qui remettent en question ce caractère impératif, tandis qu'il est impossible de séparer ces textes de l'action administrative tendant à les mettre en œuvre, tous ces éléments formant un ensemble qu'il serait artificiel de séparer.

Il est possible de dégager quelques conséquences de cette situation. Le transporteur qui respecte la règle juridique se met évidemment sous la protection de la loi. Il bénéficie alors d'une sécurité juridique. Mais celui qui l'enfreint se place dans une situation où il semble soumis à l'arbitraire: le risque d'être sanctionné est très faible, mais la sanction peut être inopinément appliquée.

L'État, en n'intervenant qu'exceptionnellement, ne modifie-t-il pas subrepticement la consistance de l'ordre juridique ? Le droit trouve le plus souvent à s'appliquer de deux façons. Il fixe les comportements autorisés, et il sanctionne ceux qui enfreignent les règles fixées. Punir ainsi avec régularité tout comportement délictueux est assurément, pour le droit, une façon d'étendre son champ d'application. La négation ou le refus de la règle juridique se trouve néanmoins saisi par le droit et la conformité à la règle posée risque d'être importante.

En ne sanctionnant qu'occasionnellement le non-respect d'une règle juridique, en quelque sorte pour l'exemple, le droit paraît limiter son champ d'application à la délimitation des comportements autorisés ${ }^{56}$. Il est du moins possible de soutenir que son mode d'intervention sur la vie sociale en est profondément affecté : l'étau se desserre mais ne disparaît pas. Des jalons sont posés, que l'exemplarité de quelques sanctions rappelle.

54. A. JEAMMAUD, « La règle de droit comme modèle ", Recueil Dalloz, 28, 1990, chron. XXXIV, p. 203, 206.

55. Ibid., p. 199 et suiv. ; P. AMSEIEK, Méthode phénoménologique et théorie du droit, Paris, LGDJ, 1964, p. 275 et suiv. ; C. MINCKE, 1998, art. cité, p. 127.

56. Il reste cependant la sanction civile, en cas de préjudice subi du fait d'une faute, en l'occurrence le non-respect d'une réglementation. Mais, en réalité, la sanction naturelle de cette réglementation est de nature pénale parce qu'elle vise avant tout à la protection d'intérêts collectifs. 


\section{S. Carré}

L'état du droit dans le transport routier de marchandises : une réglementation en trompe-l'œil
57. L'idée n'est pas neuve. Nous pensons aux positions soutenues par les représentants du "réalisme juridique " américain ou scandinave.

58. A. JEAMMAUD, « Consécration de droits nouveaux et droit positif : sens et objet d'une interrogation ", in Consécration et usages de droit nouveau, CERCRID, Université de SaintÉtienne, 1985.

\section{Conclusion}

Pour mesurer la validité d'une règle juridique, ne vaut-il pas mieux tenter de mesurer l'influence globale qu'elle a sur les relations sociales ${ }^{57}$ ? En dernière analyse, le droit ne doit-il pas être ramené à cette influence réelle, qui peut être simplement celle d'une fonction symbolique, dans la mesure où, si telle ou telle règle pourtant impérative n'est pas strictement appliquée, c'est que n'ont pas été pris les moyens légaux et matériels pour atteindre cet objectif?

En tant que phénomène social, l'observation du droit dans le secteur du transport routier de marchandises nous enseigne qu'il ne peut être limité à un ensemble de règles écrites, ni même à la jurisprudence et a $v^{\prime}$. coutumes qui viendraient se nicher dans les interstices de la loi. Ces règles ne sont qu'un point de départ pour la découverte d'une autre réalité juridique, qui est la traduction, en fonction de l'action administrative et des pratiques sociales, de cette base normative ${ }^{58}$.

Il s'agit évidemment là d'un regard extérieur, d'une constatation de sociologue et d'une prise de position théorique. Si la règle de droit est un modèle pour l'action, cela signifie que l'action va prendre en compte la règle et qu'une autre norme en résultera. Cette norme sociale se trouve être "en tension ". Elle est le produit des règles écrites et jurisprudentielles, des représentations et des pratiques autour de celles-ci. Il est difficile de dire qu'elle n'est pas au centre du phénomène juridique alors qu'elle est toujours la résultante de ces règles, qu'elle s'en rapproche ou s'en éloigne. Cette norme, une règle d'interprétation peut-on dire, devient à son tour un modèle. Chacun la mesure : les destinataires de la règle (" Jusqu'où puis-je aller, et à quel prix, tant d'un point de vue matériel que moral ? »), les praticiens du droit, l'administration, les tribunaux et le législateur lui-même qui jugera bon ou non de faire évoluer la législation en fonction de cet état du droit.

Mais d'un point de vue doctrinal, cette situation n'est évidemment pas la meilleure. Les règles de droit posées, nombreuses, mouvantes et incohérentes, gênent l'analyse dogmatique. Cette remarque ne doit cependant pas occulter le maintien nécessaire d'une présentation détaillée de ces règles, principalement dans le cadre d'un enseignement. À défaut d'être strictement respectées, ces dispositions restent le référent principal de notre droit, ce par rapport à quoi on fixera un comportement, on mesurera les risques encourus, à moins qu'on ne les invoque à l'encontre de ceux qui ne les ont pas suivies! 